\title{
LA GOBERNANZA DE LA SEGURIDAD ALIMENTARIA
}

\author{
Oliver Todt y Marta Plaza \\ Departamento de Ciencia, Tecnologia y Sociedad \\ Instituto de Filosofia, CSIC
}

\begin{abstract}
The European food safety system has recently been redesigned, following an acute loss of social acceptance, after the occurrence of several food crises on the European level. The new EU Food Law let to the creation of independent national and European food safety agencies with the participation of all concerned social actors.

This paper analyzes the recent transformation of the food safety system, including its practical application on the Spanish level. The changes in the food system are interpreted as a social management response to the general and very profound transformations in the social structure of highly industrialized societies.
\end{abstract}

KEY WORDS Governance, food safety system.
RESUMEN Debido a una profunda crisis de aceptación social, en parte por la gestión inadecuada de las diversas crisis alimentarias a nivel europeo durante los últimos 20 años, el sistema de seguridad alimentaria europeo fue completamente rediseñado. La nueva ley alimentaria, que implica la creación de agencias de seguridad alimentaria independientes, tanto a nivel europeo como español, se basa en una participación amplia de todos los actores sociales, en los principios de independencia, transparencia y excelencia, asi como en una redistribución de las competencias.

El presente artículo analiza esta transformación reciente, ofreciendo datos no sólo sobre la nueva estructura del sistema sino, además, sobre su funcionamiento en la práctica. Los recientes cambios introducidos en el sistema se interpretan como un intento de gestionar las profundas transformaciones que experimenta la estructura social de las sociedades altamente industrializadas.

PALABRAS CLAVE Gobernanza, seguridad alimentaria.

\section{Introducción: la transformación social y la gobernanza de la alimentación}

En las sociedades altamente industrializadas, se pueden observar procesos de destradicionalización e individualización que están transformando profundamente la estructura social. Los "efectos secundarios» no intencionados de la industrialización (contaminación ambiental, cambios globales y climáticos, accidentes industriales, deforestación, etc.) empiezan a convertirse en el centro de atención de los ciudadanos y enfocan su acción política (muchas veces fuera de los cauces "tradicionales" de la democracia representativa) (Todt \& González, 2006; González \& Pérez Sedeño, 2002). Dentro de este marco de transformaciones, la cuestión de la seguridad alimentaria y de su gestión cobra importancia. A causa de los cambios sociales, asi como por la creciente preocupación de los ciudadanos por los efectos de la propia industrialización, diversos aspectos de la vida diaria, tales como la alimentación, se están politizando.
Los alimentos, su industrialización acelerada en la segunda mitad del siglo XX y las consecuencias concomitantes (especialmente las "crisis alimentarias" de las últimas décadas) son, de hecho, uno de los mejores ejemplos de cómo el surgimiento de efectos no deseados o no pretendidos de la industrialización promueve su politización, convirtiendo esos mismos efectos en tema importante de la vida de los ciudadanos. Los procesos de gestión y regulación del sistema alimentario han experimentado importantes cambios en los últimos años, precisamente a causa del aumento de las preocupaciones y demandas ciudadanas. Aún más, el reciente rediseño de la legislación y del funcionamiento del sistema a nivel europeo y en los estados miembros de la Unión Europea (UE) está marcado por la introducción de conceptos característicos de la gobernanza.

El tipo de toma de decisiones que emerge dentro del sistema alimentario se puede describir como pluricéntrico, estructurado en complejas redes mediante las cuales se organizan las relaciones entre actores relativamente autó- 
nomos pero, al mismo tiempo, altamente interdependientes. A diferencia del anterior sistema, basado en coerción y control, la interrelación de los actores se caracteriza ahora en gran parte por la negociación, la cooperación, la concertación o la formación de alianzas. En otras palabras, lo importante para las decisiones son los procesos de gobernanza, más que las estructuras formales o jerarquias.

Actualmente, en respuesta a los cambios sociales, pero también a causa del surgimiento de patologías complejas (como la encefalopatía espongiforme bovina, BSE, el «mal de las vacas locas") y del desarrollo de nuevas tecnologías, se está buscando un nuevo consenso a nivel internacional sobre la gobernanza del sistema alimentario. Esta gestión tiene que abarcar no sólo cuestiones de salud humana. Se tiene que ocupar, además, de otros temas (éticos, de sostenibilidad o de biodiversidad) derivados de los efectos no pretendidos de la industrialización, que subyacen a los conflictos y preocupaciones sobre los alimentos (Todt, 2004b). Además, hay diferentes niveles de conflicto, en los que interactúan preocupaciones sobre la seguridad alimentaria con otras sobre la libertad del comercio, los derechos y libertades de los consumidores o el papel de la ciencia.

A nivel internacional se tiende hacia la creación de agencias unificadas de seguridad alimentaria que asuman la responsabilidad completa de todos los aspectos del control de los alimentos. Otro elemento común es que la labor de todas esas agencias depende de la colaboración de diversos actores sociales, como los productores y los consumidores. En muchos paises se percibe un cambio importante en la gobernanza de la seguridad alimentaria que, de estar uorientada hacia la producción", pasa a estar "orientada hacia los consumidores". Esto se refleja en las orientaciones (misiones, nivel de independencia) que se otorgan a las nuevas agencias. El modelo de un órgano único regulador que coopera y comunica estrechamente con otros actores sociales implica no sólo la creación de redes sino también nuevas formas de interdependencia.

En otras palabras, el nuevo sistema de seguridad alimentaria en la UE, con legislación y órganos reguladores de diferentes tipos, integrados pero repartidos entre distintos niveles administrativos (europeo, nacional, regional, local), da un buen ejemplo de una gobernanza multi-nivel y multiactor. Además, ninguno de los actores relevantes (organizaciones internacionales, operadores industriales y de distribución, agricultores, científicos, organizaciones no gubernamentales (ONGs), los medios de comunicación, las personas como ciudadanos y consumidores, etc.), resulta ser dominante. La gobernanza depende de todos y cada uno de estos actores, así como de su interrelación.

Todos estos actores diversos $y$, de entrada, relativamente independientes unos de otros son necesarios para que los procesos de inspección, control y regulación de los alimentos puedan funcionar. Pero también son precisos para que el sistema de seguridad alimentaria adquiera legitimidad social y esté sujeto, a su vez, a controles. En otras palabras, algunos de los actores de la red (especialmente la sociedad civil, los ciudadanos y los medios de comunicación) son necesarios para "controlar a los controladores".

\section{La alimentación: una cuestión cultural}

Los alimentos como productos de consumo presentan características que les confieren un valor único. Son productos necesarios para la vida. Pueden ser productos de consumo directo o estar elaborados, con una mezcla de ingredientes, pueden ser producidos localmente a pequeña escala (por ejemplo, para el consumo individual) o a escala industrial. Existe además una gran variedad de alimentos. Siendo un elemento fundamental de cualquier sociedad humana a nivel local, los alimentos son -al mismo tiempo- productos en un mercado globalizadado. En ese sentido, los alimentos, especialmente los que se consumen hoy en dia en los paises industrializados, pueden ser extraordinariamente complejos, porque sus diferentes ingredientes - procesados o no procesados- pueden proceder de una gran diversidad de procesos de producción y de fuentes de todas las partes del mundo.

Por todas esas razones, los alimentos presentan un problema particular, y en muchos sentidos único, de gobernanza. Aparte de la responsabilidad de las autoridades públicas de regular y controlar la seguridad, calidad y sostenibilidad de la alimentación, la gobernanza implica la participación de un gran número de actores sociales a lo largo de la cadena de producción, distribución y consumo. Por su ubicuidad e importancia, la gobernanza de la alimentación es representativa de muchas cuestiones actuales en ámbitos como las políticas públicas, la regulación y la legislación, las controversias públicas o la toma de decisiones en relación con el desarrollo científico-técnico.

La principal fuente de complejidad se deriva del hecho de que la alimentación es un concepto sociocultural. Lo que 
constituye un alimento, incluyendo posibles prohibiciones o delimitaciones de su consumo, depende del entorno cultural, pero también de opciones individuales (que, aparte de preferencias respecto a las caracteristicas organolépticas, pueden estar motivadas por razones religiosas o convicciones éticas). No existe una lista universal de alimentos "correctos». Esta situación implica que en la alimentación confluyen aspectos materiales (físicos, biológicos, etc.) con aspectos de motivación humana (cultura, psicología, sociología, etc.): la alimentación, "más que un objeto, es una interacción» (Eagleton, 1998).

Las implicaciones son evidentes: la aproximación de los consumidores a las cuestiones de riesgo en relación con los alimentos difiere de otros productos de consumo. Los riesgos relacionados con los alimentos y los efectos sobre la salud humana se suelen considerar más importantes que los riesgos relacionados con otros productos de consumo diario. Ese lugar particular ocupado por los alimentos crea un marco especial para la gobernanza de la seguridad alimentaria. Intentos, por ejemplo, de convencer a los consumidores para que adopten un determinado comportamiento por ser "mejor" -para la sociedad en general o para ellos mismosen base a resultados de investigaciones científicas sobre las propiedades de los alimentos que no tomen en cuenta otros factores sociales o culturales tienen poca probabilidad de éxito.

De hecho, en la mayoria de las sociedades existe una asociación -más o menos fuerte- entre la identidad étnica/cultural y la alimentación (ver, por ejemplo: Counihan \& Esterik, 1997; Lévi-Strauss, 1964). Hasta en la conformación del gusto puede intervenir lo cultural (Gofton \& Murcott, 2001). Para las cuestiones de gobernanza resulta además importante señalar que en diferentes aproximaciones a la alimentación (por ejemplo, la alimentación vegetariana de corte actual en los países industrializados) existe una contraposición implicita entre lo que se percibe como «natural" y lo "artificial» (de la misma forma: lo "no procesado" y lo "procesado").

En resumen, la seguridad alimentaria, operando obviamente sobre una base material, es en gran medida una construcción social (como lo demuestran muchos casos recientes, incluyendo el de los alimentos transgénicos: Muñoz, 1997, 2001, 2002; Todt \& Luján, 1997, 2000): la percepción de la seguridad de los alimentos deriva de juicios individuales sobre caracteristicas del producto (calidad) pero también sobre la manera en la que se produjo (donde intervienen, por ejemplo, consideraciones éticas, como las relativas al tratamiento de los animales, la biodiversidad o la sostenibilidad ambiental).

\section{El problema de la seguridad alimentaria}

En los últimos 30 años han tenido lugar una serie de cambios, tanto respecto a los alimentos (su composición, procedencia, procesamiento, embalaje, transporte, puesta en el mercado y consumo) como al entorno económico, social y cultural. Esos cambios han permitido ampliar la oferta, variedad y calidad de los productos alimenticios en los países industrializados. Pero, al mismo tiempo, se han revelado como fuente de nuevos riesgos.

Existe un abanico amplio de peligros y riesgos relacionados con la alimentación que incluyen, entre otros: patógenos microbiológicos, toxinas, alergénicos, ciertos aditivos intencionales o inadvertidos, productos químicos agrícolas, contaminantes ambientales, residuos de productos sintéticos utilizados en la producción de plantas y animales, consumo excesivo de determinados suplementos dietéticos, determinadas formas de preparación de los alimentos, etc. Aquí hay que tener en cuenta el aumento de la complejidad del sistema alimentario, ya que todos los agentes sociales (productores, transportadores, importadores, procesadores, distribuidores, consumidores, etc.) tienen posible influencia sobre los riesgos en diferentes puntos de la cadena.

La siguiente lista detalla las principales cuestiones que se plantean hoy en dia con respecto a la seguridad de los alimentos, así como algunos de los cambios recientes que han cobrado importancia para la gobernanza del sistema alimentario en los paises industrializados (véase también: Belton \& Belton, 2003; Phillips \& Wolfe, 2001; European Commission, 2000):

- Riesgos microbiológicos: El surgimiento de patógenos que entran en la cadena alimenticia, como Escherichia coli, el resurgimiento de patógenos ya conocidos, como Salmonella, en productos o procesos nuevos, o infecciones que se han hecho resistentes a agentes antimicrobiales.

- Contaminantes industriales y ambientales pueden afectar a la seguridad de los alimentos. Por un lado, se hace necesario el control de los productos químicos 
utilizados para la limpieza durante el procesado de alimentos, pero también de los materiales para embalajes y contenedores o de pesticidas y otros productos utilizados en la producción de plantas y animales. A causa de las preocupaciones públicas, se dedican relativamente más recursos al análisis de contaminantes químicos que microbiológicos aunque esos últimos, según muchos estudios, constituyen el problema mayor. Por otro lado, está la preocupación por la presencia de productos cancerígenos (o productos que afectan a las funciones hormonales) en los alimentos.

- Reacciones alérgicas: en su gran mayoría, se deben a diferentes tipos de nueces (incluyendo cacahuetes), huevos, leche, determinados tipos de mariscos (gambas, etc.), soja, pescado y trigo. Pero, además, hay algunos contaminantes químicos que en un porcentaje muy reducido de la población pueden causar reacciones alérgicas.

- Contaminación de productos de plantas, por ejemplo, por semillas de otro tipo o variedad, por residuos de productos químicos (herbicidas, insecticidas, etc.) o por cambios microbiales o nutricionales (aflatoxinas en maíz).

- Los productos derivados de animales pueden verse afectados por la cría inadecuada (ejemplo: la cría de pollos), la contaminación de la comida animal, residuos de hormonas o antibióticos o procesos inadecuados de producción.

- Prácticas inadecuadas de procesado, asi como procesos de producción y procesado que lleven a la introducción de microorganismos tóxicos (u otras alteraciones) o incrementen su presencia. El aumento en la complejidad de la producción de alimentos y también de la capacidad de procesado implican nuevos riesgos.

- Los principales problemas en la fase de transporte y distribución están relacionados con la mezcla inadvertida de ingredientes, equivocaciones en el etiquetado (por ejemplo, de nueces) y la contaminación o la descomposición inadvertida, especialmente en productos cárnicos y congelados.

- La distribución a nivel planetario de productos primarios y procesados incrementa los posibles riesgos, así como su dispersión. Existen riesgos vinculados a productos producidos y distribuidos localmente pero también a productos importados. La globalización del sistema alimentario convierte muchos problemas en mundiales y hace cada vez más necesaria la efectiva interacción entre actores nacionales e internacionales, políticos, científicos, sociales y económicos.

- La introducción de nuevas tecnologías cuya aplicación, además, se expande con rapidez, como los alimentos e ingredientes modificados genéticamente o la irradiación de alimentos: sus posibles efectos tóxicos o alérgicos, asi como otros efectos a largo plazo precisan de nuevas formas de evaluación de la seguridad de los alimentos $y$, potencialmente, de nuevas vías más participativas de toma de decisiones en los procesos de regulación (Luján \& Todt, 2002; Todt, 2004a).

- El aumento del consumo de alimentos con aditivos o suplementos (vitaminas, hierbas, minerales pero también, por ejemplo, edulcorantes o sustitutos de grasa) y la aparición en el mercado de alimentos funcionales hacen necesario un control de los posibles efectos de esas sustancias que normalmente no forman parte de la dieta natural o sólo en concentraciones muy reducidas. De cualquier forma, a consecuencia de las preocupaciones públicas por los efectos de productos sintéticos, muchos de esos aditivos sintéticos y sus riesgos están mejor estudiados y evaluados que sustancias que ocurren de forma natural en los alimentos.

- En los países industrializados, la estructura de consumo de muchos alimentos está cambiando. El consumo de frutas y verduras frescas está aumentando (en parte por las campañas públicas para promover una dieta más equilibrada), lo que crea una demanda durante todo el año (fuera de temporada). La importación de productos frescos en los paises industrializados crece para responder a esta demanda, pero también por los bajos costes de producción (en los paises de origen) y transporte. Eso hace necesario controles más exhaustivos de su posible contaminación.

- A causa de los recientes cambios sociales, los consumidores preparan cada vez menos comidas en casa. En cambio, aumenta el consumo de alimentos preparados, procesados y precocinados asi como el consumo de alimentos en restaurantes. Con la disminución del interés y de la capacidad de preparar comidas caseras, puede ir de la mano una disminución del conocimiento de algunas técnicas básicas de seguridad alimentaria (lavar las manos, conservación de los alimentos, etc.). Y el aumento del consumo de alimentos fuera de la casa (restaurantes, comida rápida, snacks, comida para llevar, etc.) crea otros riesgos nuevos. Al aumentar el número de trabajadores que manejan alimentos, asi como la complejidad de la preparación, se incre- 
menta el potencial para contaminaciones y transmisión de patógenos.

- Personas más susceptibles a patógenos alimenticios se ven expuestos de forma más frecuente, como muchos niños pequeños (por el incremento de comidas tomadas o preparadas fuera de casa). El aumento de la expectativa de vida de las personas, que en muchos casos va de la mano de enfermedades como el cáncer y de tratamientos médicos que reducen la capacidad del sistema inmune, tiene consecuencias para el sistema de alimentación, ya que esas personas pueden ser más susceptibles a patógenos microbiales y tener reacciones adversas a nuevos (o incluso conocidos) ingredientes alimenticios.

Pero más allá de efectos directos sobre la salud, se están produciendo cambios importantes en el entorno del sistema alimentario que afectan a su gobernanza:

- La creciente importancia económica de la industria alimentaria y la ubicuidad de los alimentos en todos los aspectos de la vida han convertido la seguridad alimentaria en una preocupación central, especialmente después de constatar los efectos económicos de las crisis alimentarias de los últimos años.

- La globalización, especialmente de los mercados pero también de las técnicas de producción, de las reglas (legislación, regulación), de los medios de comunicación, de la percepción pública, de la sociedad civil y de los valores aumenta las interacciones y los conflictos potenciales. Existen diferencias regionales importantes (por ejemplo, entre EE.UU. y la UE) que a su vez entran en conflicto con la pretensión de mercados globales y abiertos. La industria de alimentación está ya globalizada y opera a nivel internacional. La producción de alimentos se está industrializando rápidamente y a gran escala: las cadenas de producción, procesado, transporte y puesta en el mercado son cada vez más complejas y extendidas en el tiempo y el espacio. La globalización facilita el choque entre aproximaciones diferentes en la gestión y entre los diversos marcos reguladores nacionales.

- La percepción ciudadana en los países industrializados ha cambiado, a causa de la mejora del acceso a la información, de una conciencia generalmente más profunda de problemas y patologias relacionadas con la alimentación y de la experiencia reciente, especialmente en la UE, de crisis alimentarias. Un importante aspecto de ese cambio de percepción es que ahora las cuestiones de seguridad alimentaria se ven enmarcadas en cuestiones más amplias relativas al desarrollo tecnológico y a la sostenibilidad social y ambiental.

- Hay una crítica creciente de los procesos de industrialización y de sus efectos, así como de la globalización y de algunos de los actores "globalizadores» (por ejemplo, empresas multinacionales), especialmente entre sectores de la sociedad civil pero también entre la ciudadanía en general. Al mismo tiempo, la sociedad civil está creciendo, tanto al nivel local como regional, y -a su vez- globalizándose. La combinación entre la crítica de la gestión del sistema de alimentación (y de algunas de las tecnologías correspondientes) y la oposición a la globalización les da a los actores sociales críticos una fuerza y una vía de identificación adicional, además a escala planetaria. Por su relación directa con muchas cuestiones de la globalización y con el problema Sur-Norte, la gestión de la seguridad, de las nuevas tecnologías y del comercio de los alimentos (como la biotecnologia agroalimentaria) se convierte en un blanco relativamente fácil para los críticos de la globalización.

- Junto con la creciente crítica de la toma de decisiones aumenta la demanda de participación directa en las decisiones.

- La integración de los mercados significa que cualquier oposición en un mercado regional puede afectar con más facilidad que antes al mercado mundial (incluyendo los mercados de origen de las empresas que venden los productos en cuestión). Y la pérdida de confianza en una región tiende a trasladarse rápidamente a todas las regiones.

- Se constata una pérdida generalizada de la confianza de los ciudadanos en los expertos y en el conocimiento experto, en los poderes públicos y en los productores industriales. Al mismo tiempo, se puede ver un aumento de la confianza en actores de la sociedad civil (organizaciones de consumidores, ecologistas, etc.), como lo demuestran muchos estudios de percepción: European Commission, 2001, 2003; Luján \& Todt, 2000, Grove-White et al., 1997.

- Disminución de capacidad en la gestión política: la gestión política ha empezado a vincular parte de sus decisiones con (e incluso delegar en) el conocimiento científico-técnico, sea en forma de decisiones tomadas por el asesoramiento experto institucionalizado, o sea en forma del uso retórico de la racionalidad científico- 
técnica (López Cerezo et al., 1998). Está disminuyendo su capacidad de libre decisión en cuestiones relacionadas con la regulación de los sistemas tecnológicos complejos (como el de la alimentación) debido a que éstos, en muchas ocasiones, están delimitando el margen de maniobra del poder político por las realidades sociales que crean.

- Un aumento del debate (dentro de la comunidad científica, pero también a nivel público y político) sobre cómo definir, analizar, gestionar y "comunicar" conceptos como riesgo, incertidumbre o precaución. Hay, por ejemplo, tensiones entre el concepto de la «soberanía de los consumidores" y el de la uregulación basada en la ciencia", así como entre la "evaluación científica de riesgos" y los "factores no científicos" para la toma de decisiones. Aqui hay que recordar que la seguridad alimentaria puede ser más un concepto intelectual que una caracteristica biológica inherente de los alimentos. La determinación de la seguridad de un alimento incluye un juicio (necesariamente subjetivo) sobre el nivel aceptable de riesgo (basado en una evaluación de riesgo), así como la evaluación de toda una serie de valores y factores sociales.

- La introducción de nuevas tecnologías de producción de alimentos se ha convertido en un factor de riesgo para las empresas. A pesar de las fuertes inversiones públicas, su desarrollo (especialmente en el caso de la biotecnología) es, en gran parte, una empresa privada. La financiación del desarrollo de esta tecnología depende, en una medida importante, del dinero obtenido en las bolsas de valores. Esto facilita a las empresas financiar las investigaciones. Pero las convierte, al mismo tiempo, en especialmente vulnerables a los movimientos del mercado de valores, que, en gran medida, dependen de las percepciones de los inversores y de efectos psicológicos. Cualquier problema de «imagen pública" puede afectar directamente a las cotizaciones.

\section{Las personas como ciudadanos y consumidores de alimentos}

Existe una diferencia relevante entre el papel que las personas asumen como ciudadanos y el que tienen como consumidores (Muñoz et al, 2005). Esta diferencia entre papeles ejerce una influencia importante en las controversias sobre la seguridad alimentaria. Lo que las personas piden como ciudadanos no es necesariamente igual a sus prefe- rencias demostradas como consumidores de productos en el mercado. Como consumidores las personas se centran más en las caracteristicas concretas (y posibles ventajas directas y personales) del producto, mientras como ciudadanos están preocupados por la manera en la que fue elaborado y por todas las posibles consecuencias del proceso de producción, no sólo individuales, sino para la sociedad en su conjunto (efectos socioeconómicos, ambientales, etc.).

La gestión de los alimentos, consecuentemente, tiene que abarcar no sólo cuestiones de salud humana (que ha sido tradicionalmente la preocupación central del sistema de seguridad alimentaria). Se tiene que ocupar, además, de cuestiones derivadas de esos dos papeles de las personas que como consumidores valoran la calidad de los alimentos (sabor, apariencia, frescura, etc.) y como ciudadanos se preocupan por los "efectos secundarios" e impactos, individuales y colectivos (ética, biodiversidad, sostenibilidad, etc.). La gobernanza efectiva requiere un juicio sobre la importancia relativa de esas diferentes preocupaciones, tomando en cuenta la actuación colectiva de individuos como "ciudadanos", ý no sólo como "consumidores» individuales.

La conciencia ciudadana sobre cuestiones de seguridad alimentaria es actualmente más aguda en la Unión Europea que en otras partes del mundo, debido al número de recientes crisis alimentarias y a la percepción de que los intentos de gobernarlas fueron inadecuados. Esta situación se refleja en un aumento drástico de la demanda de alimentos orgánicos, así como de la presión pública para cambios en los sistemas de control y regulación.

Aun así, los estudios de percepción pública indican que el nivel general de preocupación sobre la seguridad alimentaria en diferentes paises del mundo no se diferencia mucho. Tanto en Europa como en EE.UU. una mayoria de encuestados percibe que el sistema de alimentación y especialmente el de seguridad alimentaria "no funciona muy bien" (Henson \& Traill, 2000). Pero hay diferencias importantes en los temas concretos que preocupan a los ciudadanos de distintos paises. En Europa, por ejemplo, preocupa más el uso pesticidas, hormonas y de la ingeniería genética en la alimentación; en EEUU, la irradiación de alimentos y los aditivos (Henson, 2001). En los últimos años, la cobertura mediática de cuestiones relacionadas con la seguridad de los alimentos y las crisis alimentarias ha sido significativamente más alta en la EU que, por ejemplo, en los EE.UU. Dado que se han descrito procesos de amplificación de riesgos a través 
de los medios de comunicación (Kasperson, 1992), se puede suponer que esta situación ha influido sobre la percepción pública de este tema en Europa.

También influye la falta generalizada de confianza entre los ciudadanos europeos en muchos de los actores relacionados con la producción, la distribución y la regulación de los alimentos. Los ciudadanos europeos tienden a ser escépticos en relación con la eficacia de los sistemas públicos y privados de control. Consideran que actualmente la mayor parte de la responsabilidad sobre el control de la seguridad recae en la próctica en los consumidores, aunque debería, según ellos, recaer en la industria y las autoridades públicas (que se perciben como huyendo de sus responsabilidades). Aun asi, mientras los ciudadanos estadounidenses muestran en general una mayor confianza en sus instituciones públicas, los estudios indican que el comportamiento de la industria alimentaria en EE.UU. tampoco goza de confianza (Henson \& Traill, 2000).

Respecto al comportamiento (declarado) de consumo se muestra lo que otros estudios sobre riesgo han venido demostrando desde hace tiempo (Graham \& Wiener, 1995): riesgos percibidos como bajo control del propio consumidor se aceptan con mayor facilidad. En general, los consumidores se muestran dispuestos a pagar más por obtener un nivel más alto de seguridad (por ejemplo, a través de un incremento de los controles gubernamentales). De hecho, los estudios de percepción han puesto de manifiesto que existen cuatro grupos de consumidores de alimentos que se diferencian por su principal preocupación en el momento de la compra: el grupo de los preocupados por la seguridad (casi la mitad de los encuestados), el grupo de los preocupados por la calidad, el grupo de los preocupados por el precio, y un grupo uholistico" que no tienen ninguna preocupación que destaque (Henson \& Azam, 2001).

\section{Las políticas públicas europeas en el ámbito de la seguridad alimentaria}

Después de las crisis alimentarias de los años 80 y 90 , y de la consiguiente pérdida de confianza pública, la Comisión Europea (CE) comenzó un profundo rediseño de todo el sistema europeo de seguridad alimentaria. El White Paper on Food Safety (European Commission, 2000) detalla los elementos principales de esa nueva regulación. El lenguaje de la propuesta está marcado por el enfoque en los consumi- dores y su protección. De hecho, como una de sus motivaciones principales constata la necesidad de ureestablecer la confianza pública en la provisión de alimentos, la ciencia de los alimentos y los controles de los alimentos" (European Commission, 2000: 7). Además, a pesar de incidir en la importancia suprema de la protección de la salud, destaca la importancia de las consecuencias ambientales y sociales, que -al igual que las económicas- se deben tomar en cuenta en las políticas. De hecho, a la politica ambiental se le otorga un papel fundamental en asegurar que los alimentos sean seguros.

Los principios del nuevo sistema europeo -según el White Paper- son:

1) Una aproximación integrada que abarque toda la cadena de producción, distribución y consumo.

2) La eficacia del sistema depende de la colaboración de todas las partes afectadas. La responsabilidad principal para la seguridad alimentaria recae en los productores de alimentos, asi como en los agricultores. Otros stakeholders son las autoridades competentes de los estados miembros (nacionales, regionales y locales) que vigilan el cumplimiento de la responsabilidad de los productores y la Comisión Europea que verifica y controla las capacidades de esas autoridades nacionales. $Y$, por fin, los consumidores, en los que recae la responsabilidad del tratamiento adecuado de los alimentos adquiridos.

3) La trazabilidad a través de toda la cadena de todos los productos alimenticios, de los alimentos animales y de sus ingredientes. No sólo es importante para poder reaccionar rápidamente en caso de una crisis sino también para poder identificar las fuentes de un alimento en caso de dudas sobre su seguridad.

4) Una política de seguridad alimentaria europea unificada basada en una legislación abarcadora que cubra la cadena completa de alimentación. Su desarrollo ha de ser "transparente" e incluir todas las partes afectadas. La transparencia se debe garantizar con la publicación de las opiniones científicas y de los informes de inspecciones en todo el sistema de alimentos.

5) La base de las politicas debe ser el análisis de riesgos y sus tres componentes: evaluación de riesgos, gestión de riesgos y comunicación de riesgos. El asesoramiento científico debe corresponder a los "estándares más altos" de independencia, excelencia y transparencia. Toda la legislación de seguridad ali- 
mentaria se debe basar en el asesoramiento cientifico independiente.

6) El principio de precaución se debe aplicar en la gestión de riesgos (la toma de decisiones), siempre cuando resulta ser "adecuado". Además, otros factores "legítimos" relevantes para la protección de la salud humana (como, por ejemplo, consideraciones ambientales, el bienestar animal, exigencias de los consumidores sobre la calidad de los productos, la imparcialidad de la información y la definición de las caracteristicas de los productos y métodos de producción) pueden considerarse durante la toma de decisiones.

7) Un sistema europeo integrado de recogida y procesamiento de datos que tenga capacidad de alerta rápida y que permita la identificación temprana de riesgos y peligros con el fin de prevenir situaciones de crisis.

8) Un diálogo permanente con los consumidores y la provisión de toda la información sobre el sistema de seguridad alimentaria y su operación. Los consumidores tienen el derecho de obtener información sobre la calidad de los alimentos y sus ingredientes para poder tomar decisiones informadas.

Siguiendo esas líneas, la Comisión Europea reestructuró todo el sistema europeo de seguridad alimentaria, introduciendo elementos de gobernanza (Belton \& Belton, 2003). Se redactó una ley europea unificada (food law) que regula todo el sistema alimentario (European Parliament and Council, 2002). Se creó una agencia independiente, la Autoridad Europea de Seguridad Alimentaria (European Food Safety Authority, EFSA), que centraliza la gestión y el control de todo el sistema alimentario a nivel europeo. Sus funciones abarcan proporcionar asesoramiento científico independiente, coordinar la recogida y el procesamiento de datos y llevar a cabo la supervisión del trabajo de las autoridades competentes de los estados miembros.

Todos los estados miembros crearon, a su vez, agencias similares. En España se creó la Agencia Española de Seguridad Alimentaria, AESA, con estatus de organismo autónomo de la administración Estatal (Reino de España, 2001; 2002). La legislación europea aspira a que todas esas agencias funcionen sobre la base de la independencia de sus respectivos ejecutivos y que tengan como objetivos operacionales garantizar la transparencia, la excelencia e independencia en sus informes científicos, asi como permitir la participación de todos los actores sociales relevantes en la toma de decisiones. De hecho, tanto la EFSA como la AESA incluyen la participación de diversos agentes sociales (también de la sociedad civil) en sus órganos de gestión y asesoramiento, tienen políticas de acceso público a la información e intentan dar garantías de su independencia mediante declaraciones de conflictos de intereses de los miembros de sus comités científicos y sus altos cargos.

\section{La European Food Safety Authority}

La Agencia Europea de Seguridad Alimentaria (European Food Safety Authority, EFSA) se convierte en el nuevo sistema regulador en el foco principal de todas las actuaciones, tanto a nivel comunitario como a nivel de los estados miembros (incluyendo sus agencias nacionales o regionales). A continuación se detallan algunos de los elementos principales de la EFSA.

- La misión de la EFSA se define con amplitud, debiendo tener en cuenta, por ejemplo, cuestiones relativas al bienestar de los animales o a los efectos ambientales de la producción de alimentos. También es responsable de generar opiniones científicas sobre los organismos modificados genéticamente (OMGs), incluyendo productos transgénicos que no sean alimentos.

- Las funciones concretas de la EFSA son la preparación y provisión de asesoramiento científico (especialmente a la Comisión Europea), la recogida, coordinación y análisis de la información necesaria, la vigilancia y el monitoreado de desarrollos relevantes para la seguridad alimentaria, incluyendo la alerta rápida, y la comunicación de sus resultados a todas las partes interesadas.

- Independencia, excelencia científica y transparencia en su funcionamiento. La EFSA debe "estar guiada por la mejor ciencia posible, ser independiente de intereses industriales y políticos, estar abierta a un control público riguroso, estar acreditada científicamenten (European Commission, 2000). Aún así, debe haber un balance apropiado entre independencia, por un lado, y responsabilidad y representatividad (de todos los puntos de vista relevantes), por otro. La excelencia se conseguiria mediante el alistamiento de los mejores científicos independientes. La transparencia implicaria la publicación rápida de todos los resultados, recomendaciones y procedimientos abiertos.

- El objetivo de la EFSA es "contribuir a un alto nivel de protección de la salud de los consumidores en el área 
de la seguridad alimentaria, con el fin de reestablecer y mantener la confianza de los consumidores." Su misión estará guiada "principalmente por el interés públicon (European Parliament and Council, 2002: 3). De hecho, reestablecer y mantener la confianza de los europeos mediante una protección efectiva de los consumidores y su salud es el objetivo más reiterado de la nueva legislación. La función de la EFSA de ser una fuente cientifica independiente de asesoramiento, información y comunicación de riesgo sirve, en primer lugar, para "mejorar la confianza de los consumidores" (European Parliament and Council, 2002: 3). Una parte implicita de su misión es convertirse en el punto de contacto uúnico y visible» para todas las partes afectadas. En otras palabras, el objetivo final es que la EFSA sea, de forma cuasi automática, el primer y único punto de contacto para todos los actores si precisan de información científica sobre temas o problemas relacionados con la seguridad alimentaria.

- La EFSA tiene que ser extremadamente responsable en la rendición de cuentas (accountability) ante las instituciones europeas y los ciudadanos.

- Separación clara entre evaluación del riesgo (función de la EFSA) y gestión del riesgo (función de la Comisión Europea). La EFSA es responsable de la evaluación y la comunicación de riesgo. La evaluación de riesgo se traduce en la provisión de asesoramiento científico. La comunicación de riesgo implica la diseminación rápida de toda la información, pero también reducir las posibilidades de que se generen preocupaciones "inadecuadas" sobre la seguridad de los alimentos (ver siguiente punto). En cambio, la autoridad de la decisión estará en manos de la Comisión Europea. Este reparto de competencias se justifica con la necesidad de que las decisiones sean tomadas por un órgano sujeto al control democrático. Pero también abre la puerta a que la Comisión considere en sus decisiones informaciones de otras fuentes que no sean la EFSA: la regulación estipula que la legislación alimentaria y todas las medidas en relación con la seguridad alimentaria "generalmente deberian basarse en el análisis de riesgo, excepto en situaciones en las que eso no resulta adecuado debido a las circunstancias o el tipo de medidas contempladas" (European Parliament and Council, 2002: 8; cursivas nuestras). En otras palabras, "otros factores" (sociales, económicos, tradicionales, éticos y ambientales) pueden fundamentar las decisiones.
- La EFSA asume la responsabilidad de la comunicación del riesgo, independiente de la CE. Según la regulación, la agencia debe confeccionar información "objetiva, solvente y fácil de comprender" y diseminarla rápida y activamente entre todos los actores implicados. La comunicación del riesgo en si se define como uinteractiva", como un "intercambio de información entre las partes afectadas», en forma de un "diálogo con y feedback desde todos los actores relevantes». Para eso, es necesario que las decisiones sean utransparentes y públicas" y que las preocupaciones de los consumidores se tengan en cuenta mediante consultas públicas sobre todos los aspectos de la seguridad alimentaria, audiencias públicas entre expertos y consumidores, así como la facilitación del intercambio entre consumidores a nivel europeo. La transparencia llevará a la "supervisión pública necesaria" y garantizará el control democrático. Por otro lado, se debe garantizar que los consumidores puedan tomar decisiones informadas a base de información suficiente sobre cada producto, su composición, origen y métodos de producción (garantia del etiquetado comprensivo).

- El principio de precaución se reconoce explicitamente en la regulación. En situaciones de incertidumbre cientifica, cuando existe riesgo para la vida o la salud, las decisiones se pueden basar en ese principio. La única restricción expresa es que debe aplicarse según una base común en toda la UE. Las medidas adoptadas deben ser "proporcionales" y "restringir el comercio sólo en la medida necesaria» (European Parliament and Council, 2002: 9). La precaución se operacionaliza como «medidas provisionales de gestión de riesgo, ... a la espera de información científica adicional que permita una evaluación de riesgo más completon. En otras palabras, la definición de la precaución adoptada lleva implicita la idea de poder resolver el problema en cuestión con más información científica, confundiendo asi los conceptos de incertidumbre, ignorancia e indeterminación (Wynne, 1992).

- Todos los miembros de la EFSA, incluyendo los miembros de los Paneles y del Comité Científico, deben actuar «de forma independiente" y "para el interés público" (European Parliament and Council, 2002: 18). Para garantizar su independencia, deben hacer una declaración anual y pública de intereses y declarar posibles conflictos de interés en cada reunión a la que asisten.

- Debe haber un acceso público «amplion a todos los documentos en poder de la EFSA. La EFSA debe hacer 
públicas «inmediatamente» las actas de las reuniones de los Paneles y del Comité Científico, así como sus opiniones (incluyendo opiniones minoritarias) y las informaciones utilizadas para establecer esas opiniones.

- El Consejo Administrador de la EFSA, que elige el director ejecutivo de la agencia, debe contar con cuatro (sobre un total de 14) personas provenientes de organizaciones que representan a los consumidores y otros intereses de la cadena alimentaria. La coordinación con las agencias de los estados miembros se garantiza mediante un Foro Asesor, compuesto por las autoridades competentes nacionales.

La Regulación se ocupa reiteradamente del tema de la autoconsistencia interna del asesoramiento científico y establece reglas cuasi-administrativas para intentar llegar a clausura en el caso de divergencias científicas. El mecanismo intenta prevenir informes divergentes y establecer la EFSA como autoridad científica única. La EFSA debe identificar potenciales divergencias entre sus pareceres cientificos y los de otros órganos similares. Si el otro órgano pertenece a la administración europea o la de un estado miembro (por ejemplo, una agencia nacional de seguridad alimentaria), está obligado a cooperar con la EFSA para resolver la divergencia o para presentar un documento conjunto (que se debe hacer público) explicando las divergencias cientificas e identificando las incertidumbres existentes en los datos científicos (véase el apartado 8 de este artículo).

\section{La implementación de la gobernanza en la seguridad alimentaria en España}

Mediante la puesta en funcionamiento, en el año 2002, de la Agencia Española de Seguridad Alimentaria (AESA), se está imponiendo también en España un nuevo modelo de gestión del sistema alimentario que se caracteriza por el concepto de la gobernanza.

Los tres principios fundamentales de actuación de la Agencia europea EFSA se ven reflejados también en la legislación que establece la AESA como organismo autónomo de la administración Estatal, adscrita al Ministerio de Sanidad y Consumo. La actuación de la Agencia debe seguir los principios de "transparencia e independencia" y sus decisiones deben estar basadas en evaluaciones científicas urigurosa[s] y de excelencian (Reino de España, 2001).
La AESA tiene la función básica de "ofrecer garantías e información objetiva a los consumidores y agentes económicos del sector agroalimentario español». De hecho, se invoca la "participación activa" de los consumidores, los operadores económicos y sociales, así como de la comunidad cientifica, en el funcionamiento de la AESA, aunque la operacionalización de esa participación la establece la práctica (véase abajo).

La AESA tiene como objetivo generar conocimiento cientifico para la toma de decisiones. Pero a diferencia de la EFSA (cuya misión se limita expresamente a la evaluación y la comunicación del riesgo, mientras la Comisión Europea se reserva la toma de decisiones), también tiene capacidad de decisión.

En el Consejo Administrador de la EFSA, cuatro de los 14 miembros representan a la sociedad civil y la industria. En cambio, en el Consejo de Dirección de la Agencia Española (que, incluyendo a la presidenta, tiene 15 miembros, de ellos seis directamente nombrados por diferentes ministerios, incluyendo los dos vicepresidentes), sólo hay un representante de las organizaciones de consumidores (a través del Consejo del Consejo de Consumidores y Usuarios) y un representante del sector económico.

Sin embargo, a diferencia de la Agencia Europea, la AESA tiene un Consejo Consultivo (con reducidas atribuciones formales, que se limitan principalmente a su consulta para el programa de actividades y a recibir información en situaciones de crisis), en el que, a parte de la presidenta, figuran seis representantes de la sociedad civil, tres de asociaciones de consumidores y tres propuestos por el Consejo Económico y Social; ocho representantes de organizaciones económicas; y seis representantes de organizaciones colegiales (médicos, farmacéuticos, veterinarios, ingenieros agrónomos, biólogos y químicos).

Los primeros datos sobre el funcionamiento del sistema español en la práctica (basados en un estudio cualitativo: Plaza, 2004) indican que para la mayoria de los actores sociales implicados, la implantación del nuevo sistema ha supuesto un salto cualitativo, un cambio en el que los aspectos más valorados por los actores del sistema han sido el aumento de la participación, la apertura a la comunicación mostrada por la AESA, así como el suministro de información regular por parte de la Agencia. Se percibe la gestión como proactiva y ágil, al menos en la rutina, ya que no 
se han producido grandes crisis que permitan evaluar la gestión de este extremo. Los nuevos actores invitados al sistema, especialmente las organizaciones de consumidores y los sindicatos, se encuentran bastante satisfechos con el mismo, aunque cuentan con la limitación de su escasa experiencia en este tipo de gestión participativa. Por esta razón, aún no han ejercido una labor de formulación proactiva de demandas, limitándose en un principio a "ser informados".

Existen varios aspectos que suscitan duda entre los participantes, entre ellos, el nivel de independencia de AESA. Por ejemplo, dada la ausencia de situaciones de crisis, la independencia en la actuación de la Agencia del gobierno Estatal todavia no se ha podido valorar. Otro punto de crítica es el nivel de financiación del Comité Científico de la AESA, considerado demasiado bajo. Sin suficientes medios, corre el riesgo de hacer evaluaciones de riesgos basadas fundamentalmente en revisiones bibliográficas, ante la imposibilidad de realizar estudios propios con la calidad suficiente. El conflicto más importante está relacionado con el acceso a la información. Algunas de las organizaciones de consumidores han reclamado acceso a los informes completos del Comité Científico de la Agencia, incluyendo posibles posiciones divergentes, para que puedan conocer las incertidumbres científicas presentes en las deliberaciones del Comité, y no sólo el informe final consensuado. La AESA se niega a hacer pública esa información porque la ley no le obliga a ello.

En definitiva, el nuevo sistema de gobernanza de la seguridad alimentaria en España significa un cambio radical en la regulación, entre otras cosas, porque por primera vez se implementa una participación sistemática de actores de la sociedad civil. Ahora bien, la reducida representación de la sociedad civil en la AESA y la falta de experiencia de las propias organizaciones de consumidores en la gestión participativa levantan dudas sobre su capacidad de trasladar las inquietudes reales de los consumidores a las estructuras de decisión de la Agencia. Los conflictos sobre el acceso a los datos científicos, a pesar de un nivel de transparencia relativamente alto en el funcionamiento de la AESA, indican que sólo la práctica reguladora mostrará si este nuevo sistema será capaz de ganar y mantener la confianza de los ciudadanos españoles.

\section{El papel del conocimiento científico}

La introducción de procesos de gobernanza también tiene consecuencias para el papel del conocimiento científico especializado en la toma de decisiones (López Cerezo \& González, 1993). Los anteriores procesos de regulación de la ciencia y la tecnología basaban sus decisiones generalmente en expertos, asi como en una ciencia adaptada a la toma de decisiones reguladoras (la ciencia reguladora: López Cerezo \& Luján, 2000; Luján \& Echeverría, 2004). Pero en el nuevo sistema europeo de seguridad alimentaria, a pesar del papel destacado que tiene la ciencia para proporcionar datos, su papel resulta ambivalente.

Según la nueva ley alimentaria europea, en la toma de decisiones, que depende de instituciones políticas legitimadas por elecciones, pueden intervenir "otros factores", aparte de los datos científicos. Además, como se ha expuesto anteriormente, mientras la Agencia Europea de Seguridad Alimentaria está encargada de la evaluación del riesgo, la Comisión Europea se reserva expresamente la gestión del riesgo (toma de decisiones). De esta manera, se define la gobernanza de la seguridad alimentaria como un proceso apoyado por evaluaciones y metodologías científicas pero no basado (exclusivamente) en ellas. En cierto modo, por primera vez de esa forma tan explícita la regulación europea del sistema alimentario cimienta un nuevo rol de la ciencia para la toma de decisiones políticas.

Con la food law europea, se urelegan la ciencia formalmente al puesto de una fuente de información (entre varias) para la toma de decisiones. En otras palabras, se hace constar explicitamente la supremacía de la capacidad de decisión politica sobre la aproximación cientifica. Aunque en la práctica las decisiones políticas sigan utilizando los resultados científicos como base y justificación, la ley da mucho margen de maniobra a los que toman las decisiones a nivel politico.

Es importante resaltar que la principal novedad es uretóricail, no práctica. En todos los sistemas de regulación anteriores, se pueden encontrar casos en los que las necesidades políticas toman precedencia sobre las afirmaciones científicas. Pero con la nueva ley alimentaria, la Comisión Europea abandona la retórica de la ciencia como fuente única de las decisiones: a consecuencia de las críticas públicas y de la pérdida de confianza, en la retórica la ciencia es fuente ya sólo de información relevante pero no base única de las decisiones. En eso compite ahora con, por ejemplo, el principio de precaución (aunque para él también se invoque una base científica) o con los "otros factores" (sociales, ambientales, éticos, etc.) relevantes que menciona la ley. En

ARBOR CLXXXI 715 SEPTIEMBRE-OCTUBRE (2005) 403-416 ISSN: 0210-1963 
EE.UU., en cambio, la ciencia sigue siendo el foco retórico principal de la regulación de la ciencia y la tecnología, y el único factor en el que, según las leyes, se deben basar las decisiones.

Aún así, la práctica de la toma de decisiones en la UE muestra que las decisiones pueden seguir basándose en la ujustificación científican (como en el caso de la autorización de los organismos modificados genéticamente). De la misma forma, hay casos documentados en los que los órganos reguladores estadounidenses utilizaron otros factores, aparte de la ciencia, en sus decisiones (Busch, 2002). De hecho, parece que en la práctica reguladora, las autoridades de la UE y de EE.UU. no se diferencian mucho en la aplicación de los diferentes principios de decisión (resultados científicos, precaución, familiaridad, etc.), sólo que los reguladores europeos o estadounidenses los aplican a diferentes ámbitos, según determinadas necesidades, intereses o factores culturales.

Otra novedad de la food law es que la regulación europea de seguridad alimentaria reconoce explicitamente las divergencias dentro de las propias comunidades científicas. De hecho, como ya vimos, define por ley un proceso de unificación de los pareceres científicos en caso de que hubiera divergencias entre las diferentes instituciones europeas (por ejemplo, entre la EFSA y una de las agencias nacionales). Según la situación, este proceso se reviste de closure forzada (si hay posibilidad de unificar los pareceres) o de aclaración pública de las divergencias (si a pesar de todos los intentos las diferentes instituciones no se ponen de acuerdo). En este último caso, la ley obliga a la publicación de las diferentes posturas, incluyendo una explicación de las divergencias y de las incertidumbres. En otras palabras, también en este caso el tratamiento de la ciencia es ambivalente: por un lado, se intenta conseguir una homogeneización de la ciencia para la regulación, su metodología y sus resultados (con el fin de reforzar la autoridad de los pareceres cientificos de la EFSA). Por otro, se permitiría a los ciudadanos contemplar abiertamente la ruptura dentro del conocimiento científico (en caso de publicar divergencias entre órganos europeos sin posibilidad de resolver) y ser informados, con "exactitud científica", sobre las incertidumbres existentes que hacen imposibles la closure.

En resumen, se puede observar un papel distinto de la ciencia en la toma de decisiones reguladoras, más ambiguo que en el sistema de seguridad alimentaria anterior, por lo menos en la retórica. Esto es otro ejemplo de cómo la función de la ciencia para la regulación está cambiando, al aumentar el cuestionamiento de la ciencia como base privilegiada para las decisiones, a consecuencia del traslado de las controversias científicas (normales) hacia el ámbito público y por las implicaciones que tienen para la toma de decisiones (Echeverría, 2003). Estos cambios en la función de la ciencia para los procesos de regulación se unen a la aparición de una cierta "bifurcación" dentro de la propia ciencia (no sólo reguladora sino también académica): por un lado, las ciencias de laboratorio "clásicas" y las ingenierías que impulsan el desarrollo científico-técnico pero que cada vez pueden contribuir menos a la solución de los problemas de gestión; y por otro lado, las ciencias "postnormales» que son directamente relevantes para el control de los efectos no deseados producidos por la ciencia y la tecnología (Ravetz, 2003: 824). 0, en otras palabras, por un lado, las ciencias y tecnologias que contribuyen a la modernización y profundización de la industrialización a nivel mundial; $y_{1}$ por otro, las ciencias más recientes de "limpieza y supervivencian (Ravetz, 2002: 258), menos maduras en su penetración y estructuración social, así como en su aparato teóri-

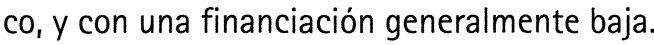

\section{Conclusiones}

La gobernanza del sistema alimentario es un caso ilustrativo del ámbito político surgido a raíz de la modernización reflexiva, en el que se expresa actualmente la acción civica de los ciudadanos. El sistema de seguridad alimentaria, a nivel europeo y español, fue rediseñado radicalmente en los últimos años, en parte para dar respuesta a una serie de demandas ciudadanas y transformaciones en la estructura de las sociedades altamente industrializadas. Con el fin de ganar y mantener la confianza de los ciudadanos (requisito clave para el actual desarrollo científico-tecnológico) en los procesos de regulación, se instauró un sistema más independiente de los respectivos ejecutivos, se aumentó la transparencia y el acceso a la información y se permitió la participación social de forma relativamente amplia.

Este caso muestra cómo la cuestión de la confianza de los ciudadanos en los sistemas de toma de decisión se está convirtiendo en uno de los temas fundamentales del desarrollo tecnológico contemporáneo. Además indica cómo en una situación de globalización económica, que prima la apertura de los mercados mundiales (lo que en principio podria 
beneficiar a los grandes operadores multinacionales), el "poder ciudadano" puede aumentar: crece la necesidad de mantener la confianza de los ciudadanos y consumidores en los productos y su proceso de regulación. De hecho, los cambios introducidos en la gestión del sistema alimentario europeo se pueden interpretar como un intento de gestionar las transformaciones en la estructura social de las sociedades altamente industrializadas y sus efectos para el desarrollo científico-tecnológico.

También en España se ha dado un cambio drástico en la toma de decisiones, con la introducción de procesos más participativos, transparentes y accesibles. Pero, al mismo tiempo, los actores perciben limitaciones y expresan dudas sobre el alcance real de esos cambios, especialmente en cuanto a su eficiencia y su capacidad de generar y mantener la confianza pública.
Hay que resaltar que la introducción de los procesos de gobernanza en España está impulsada en gran medida por las exigencias de la propia legislación europea. Mientras a nivel europeo, la gobernanza se puede interpretar como una respuesta a las exigencias sociales y como resultado de un proceso de aprendizaje social, en España las exigencias desde la sociedad civil son casi inexistentes, las organizaciones de consumidores están poco preparadas y la sociedad civil en general resulta ser débil y desorganizada (López Cerezo \& González, 2002; Todt, 1999). En esta situación, la introducción de la gobernanza por mandato legal europeo podría tener el efecto paradójico de poner en marcha un proceso de aprendizaje social en España (en vez de ser su resultado), principalmente porque obliga a actores sociales anteriormente aislados de entrar en contacto, comunicación y negociación.

\section{BIBLIOGRAFÍA}

Belton, P.S. \& Belton, T. (eds.) (2003): Food, Science and Society. Heidelberg: Springer.

Busch, L. (2002): The Homiletics of Risk, Journal of Agricultural and Environmental Ethics, 15, 17-29.

Counihan, C. \& Esterik, P. (1997): Food and culture. New York: Routledge.

Echeverria, J. (2003): La revolución tecnocientifica. Madrid: Fondo de Cultura Económica de España.

Eagleton, T. (1998): Edible ecriture, en: S. Griffiths \& J. Wallace (eds.), Consuming passions, London: Mandolin, 203-208

European Commission (2000): White Paper on Food Safety, COM(1999) 719 final. Brussels: European Commission

European Commission (2001): Eurobarometer 55.2: Europeans, science and technology. Luxembourg: European Commission.

European Commission (2003): Eurobarometer 58.0: Europeans and Biotechnology in 2002. Luxembourg: European Commission.

European Parliament and Council (2002): Regulation (EC) No 178/2002 of the European Parliament and of the Council of 28/1/2002, laying down the general principles and requirements of food law, establishing the European Food Safety Authority and laying down procedures in matters of food safety, Official Journal of the European Communities, L 31 , 1/2/2002.
Gofton, L. \& Murcott, A. (2001): The special place of chocolate in the AngloAmerican diet: towards a sociology of food cravings and addictions, en: $M$. Hetherington (ed.), Food cravings and addictions, Leatherhead: Leatherhead International

González, M.I. \& Pérez Sedeño, E. (2002): Ciencia, tecnología y género, Revista Iberoamericana de Ciencia, Tecnología, Sociedad e Innovación, n 2-2002.

Graham, J.D. \& Wiener, J. B. (eds.) (1995): Risk versus Risk. Cambridge: Harvard University Press.

Grove-White, R. et al. (1997): Uncertain World. Genetically Modified Organisms, Food and Public Attitudes in Britain. Lancaster: Centre for the Study of Environmental Change - Lancaster University

Henson, S. (2001): Consumer Perceptions of Food Safety, en: P. Phillips \& R. Wolfe (eds.), Governing Food. Montreal: McGill-Queen's Univ. Press.

Henson, S. \& Azam, M. (2001): Trade-off between Food Safety and other Product Attributes in Food Choice. Reading: University of Reading.

Henson, S. \& Traill, W. (2000): Measuring Perceived Performance of the Food System and Food-Related Welfare, Journal of Agricultural Economics, 51(3), 388-404.
Kasperson, R.E. (1992): The Social Amplification of Risk, en: S. Krimsky \& D. Golding (eds.), Social Theories of Risk, Westport: Praeger.

Lévi-Strauss, C. (1964): The raw and the cooked. London: Cape.

López Cerezo, J.A. \& González, M.I. (1993), The Role of Technical Expertise in Policy Implementation, Technology in Society, 15, 383-397.

López Cerezo, J.A. \& González, M.I. (2002): Política del Bosque: expertos, políticosy ciudadanos en la polémica del eucalipto en Asturias. Madrid: Cambridge University Press/OEI.

López Cerezo, J.A. \& Luján, J.L. (2000): Ciencia y política del riesgo. Madrid: Alianza.

López Cerezo, J.A., Méndez, J.A. \& Todt, 0. (1998): Participación pública en política tecnológica: problemas y perspectivas, Arbor, no. 627, 279-308.

Luján, J.L. \& Echeverria, J. (eds.) (2004): Gobernar los riesgos. Madrid: Biblioteca Nueva/ OEI.

Luján, J.L. \& Todt, 0. (2000). Perceptions, attitudes and ethical valuations: the ambivalences of the public image of biotechnology in Spain, Public Understanding of Science, 9, 383-392. 
Luján, J.L. \& Todt, O. (2002): Dinámica de la precaución. Sobre la influencia de los conflictos sociales en la regulación de los OGMs, en E. lañez (ed.), Plantas transgénicas: de la ciencia al derecho, Granada: Comares.

Muñoz, E. (1997): Nueva biotecnología y sector agropecuario. El reto de las racionalidades contrapuestas. IESA documento de trabajo 97-02. Madrid: IESA.

Muñoz, E. (2001): Biotecnologia y sociedad. Madrid: Cambridge Univ. Press.

Muñoz, E. (2002): La cultura científica, la percepción pública y el caso de la biotecnología, Documento de trabajo 02-07. Madrid: UPC. [http://www.iesam.csic.es/doctrab.htm].

Muñoz, E., Plaza, M., Ponce, G., Santos, D. \& Todt, 0. (2005): La opinión de los consumidores españoles sobre los alimentos transgénicos y su seguridad, Revista Internacional de Sociologia, $n^{\circ}$ 41 (2/05), 93-108.

Phillips, P. \& Wolfe, R. (eds.) (2001): Governing Food. Montreal: McGillQueen's Univ. Press.

Plaza, M. (2004): Percepción del nuevo sistema de gestión de la seguridad alimentaria - Documento de Trabajo IF/FECYT 1205. Madrid: IF-CSIC.

Ravetz, J.R. (2002): Food Safety, Quality, and Ethics - A Post-Normal Perspective, Journal of Agricultural and Environmental Ethics, 15, 255-265.

Ravetz, J.R. (2003): A paradoxical future for the safety in the global knowledge economy, Futures, 35, 811-826.

Reino de España (2001): Ley 11/2001, de 5/7/2001, por la que se crea la Agencia Española de Seguridad Alimentaria, BOE, no. $161,6 / 6 / 2001$

Reino de España (2002): Real Decreto 709/2002, de 19/7/2002, por la que se abrueba el Estatuto de la Agencia Española de Seguridad Alimentaria, BOE, no 178, 26/7/2002.

Todt, 0. (1999): Social Decision Making on Technology and the Environment in Spain, Technology in Society, 21, 201216.

Todt, 0. (2003): Designing trust, Futures, 35, 239-251.

Todt, 0. (2004a): Regulating agricultural biotechnology under uncertainty, Safety Science, 42, 143-158.
Todt, 0. (2004b): El conflicto sobre la ingeniería genética, y los valores subyacentes, Sistema, no 179-180, 89102.

Todt, O. \& Luján, J.L. (1997): Labeling of Novel Foods, and Public Debate, Science and Public Policy, 24 (5), 319-326.

Todt, 0. \& Luján, J.L. (2000). Spain: commercialization drives public debate and precaution, Journal of Risk Research, 3 (3), 237-245.

Todt, 0. \& González, M. (2006): Del gobierno a la gobernanza: nuevas dimensiones de la política sobre ciencia y tecnología, Isegoría, en prensa

Wynne, B. (1992): Uncertainty and Environmental Learning, Global Environmental Change, June 1992, 111-127. 